\title{
Parent-child transmission of infantile cholestasis with lymphoedema (Aagenaes syndrome)
}

\author{
Andrew A M Morris, J Silvia S Sequeira, Marion Malone, Sarah F Slaney, \\ Peter T Clayton
}

The Institute of Child Health, 30 Guilford Street, London WC1N 1EH, UK

A A M Morris

J S S Sequeira

M Malone

S F Slaney

P T Clayton

Correspondence to: Dr Clayton.

Received 16 November 1996 Revised version accepted for publication 3 April 1997

\begin{abstract}
We report a mother and daughter with features of Aagenaes syndrome. Unlike most previous cases, there is no Norwegian ancestry and the pedigree favours dominant rather than recessive inheritance.

$(\mathcal{F}$ Med Genet 1997;34:852-853)
\end{abstract}

Keywords: Aagenaes syndrome; cholestasis; lymphoedema

Infantile cholestatic liver disease with subsequent lymphoedema (Aagenaes syndrome, McKusick 214900) has been reported in four Norwegian pedigrees $^{1-3}$ and one Italian patient. ${ }^{4}$ In all but the latter, jaundice started in the neonatal period and resolved during childhood, though cholestatic episodes recurred during adult life. Lymphoedema of the legs appeared during infancy in one pedigree and always before puberty. The aetiology is un-

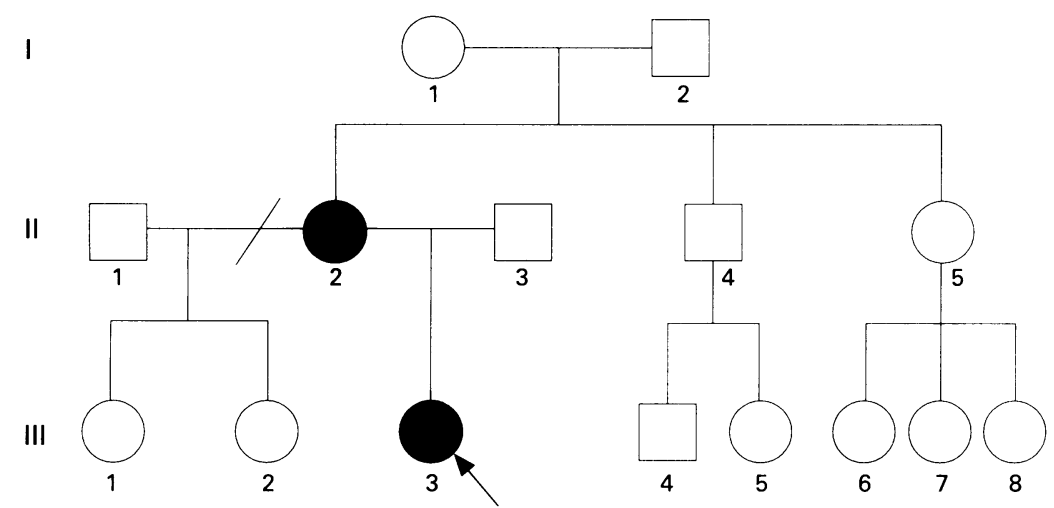

Figure 1 Pedigree of the family.
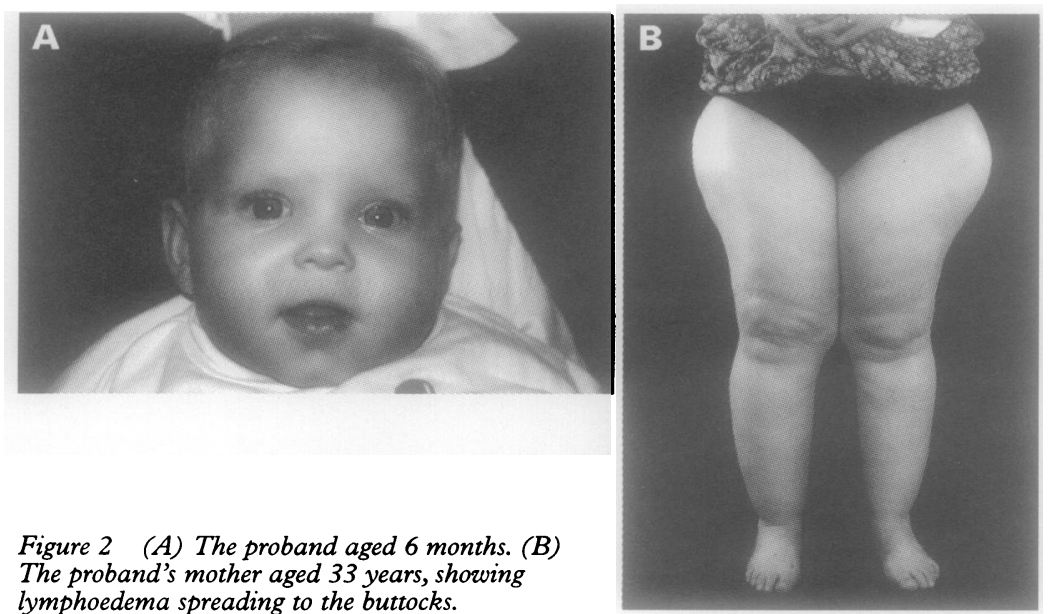

Figure 2 (A) The proband aged 6 months. (B) The proband's mother aged 33 years, showing lymphoedema spreading to the buttocks. known but autosomal recessive inheritance has been suggested.

\section{Case report}

Our proband (III.3, fig 1) was born at 36 weeks' gestation. Jaundice was noted from the age of 3 days, worsening at 6 weeks, with pale stools and dark urine. The liver was enlarged to $3 \mathrm{~cm}$ below the costal margin and the spleen tip was palpable. There was no dysmorphism (fig 2A) nor any abnormalities on ophthalmological examination, echocardiography, or spinal $x$ rays. Conjugated hyperbilirubinaemia was accompanied by markedly raised levels of bile acids, transaminases, and alkaline phosphatase. A HIDA scan showed prompt liver uptake but no passage of isotope into the bowel. A liver biopsy containing seven portal tracts showed a complete absence of interlobular bile ducts, with secondary proliferation at the marginal plate (fig 3). The portal tracts contained a mild lymphocytic infiltrate without increased fibrosis. There was marked cholestasis within lobules and giant cell transformation with occasional acidophil bodies. The patient was treated with phenobarbitone and fat soluble vitamins. At 1 year of age, the total bilirubin is $20 \mu \mathrm{mol} / 1$ and there is still no lymphoedema.

The proband's mother (II.2, fig 1) was born at 32 weeks' gestation, labour having been induced because of pre-eclampsia. There were no neonatal problems but increasing jaundice was noted from 6 weeks with mild hepatosplenomegaly. Ascending cholangiography was normal but a liver biopsy showed identical changes to those in her daughter. None of the 23 portal tracts examined contained bile ducts. The jaundice gradually improved but she has suffered persistent itching, which worsened during her pregnancies. From 11 years of age, she developed progressive lymphoedema despite normal plasma protein concentrations. At 33 years, she has normal liver function but lymphoedema has spread to her buttocks and lower abdomen (fig 2B) and she has swollen arms. Ophthalmological examination showed posterior embryotoxon but echocardiography and spinal $x$ rays were normal. The proband's parents are non-consanguineous, as are the maternal grandparents. There is no known Norwegian ancestry.

\section{Discussion}

The findings in this family correspond to Aagenaes syndrome, confirming that the disorder occurs in non-Norwegian families. Analysis of previous pedigrees suggests autosomal 


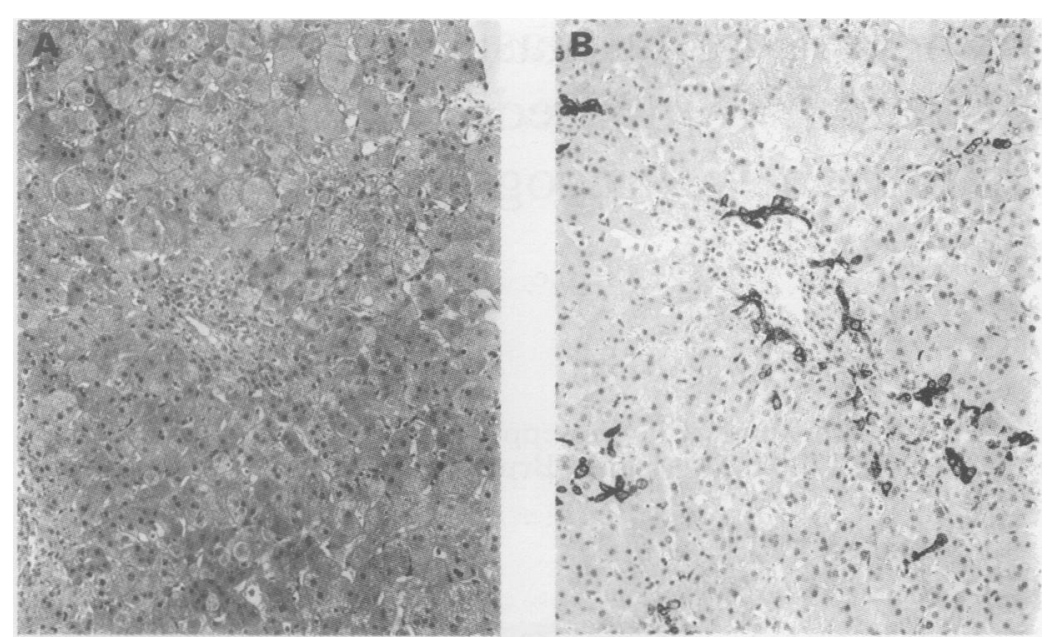

Figure 3 Serial sections of liver from the proband aged 8 weeks $(A)$ stained with $H \mathcal{F} E$ and $(B)$ immunostained for low molecular weight cytokeratin, which selectively stains bile duct epithelial cells. The main interlobular bile duct is absent, with secondary proliferation of bile ducts at the margin of the portal tract.

recessive inheritance; most patients had affected sibs and normal parents, with a high frequency of consanguinity. There has been one previous instance of mother-child transmission, but as the father came from the same isolated community and may have been a het- erozygote, this was probably an example of pseudodominance. ${ }^{1}$ By contrast, in our family the parents come from different regions of the $\mathrm{UK}$, where the condition is very rare.

There are two possible explanations for the mother-child transmission in our family. Firstly, the mother could have two recessive mutations, the proband being a manifesting heterozygote (or vice versa). This possibility is favoured by another pedigree, in which the father, uncle, and paternal grandmother of an affected child had plantar lymphangiomata. ${ }^{2}$ Alternatively, Aagenaes syndrome in our family may be caused by an autosomal dominant mutation arising de novo in the mother; this is the explanation we consider most likely. Such a mutation might be allelic with those in previous patients or Aagenaes syndrome may result from defects in several different genes.

1 Aagenaes $\emptyset$, Van der Hagen CB, Refsum S. Hereditary recurrent intrahepatic cholestasis from birth. Arch Dis Child 1968;43:646-57.

2 Sharp HL, Krivit W. Hereditary lymphedema and obstructive jaundice. $\mathcal{F}$ Pediatr 1971;78:491-6.

3 Aagenaes $\emptyset$. Hereditary recurrent cholestasis with lymphoedema - two new families. Acta Paediatr Scand 1974;63: 465-71.

4 Vajro P, Romano A, Fontanella A, Oggero V, Vecchione R, Shmerling DH. Aagenaes syndrome in an Italian child. Acta Paediatr Scand 1984;73:695-6. 